\title{
Meson correlators in the $\varepsilon$-regime of two-flavor lattice QCD
}

\author{
JLQCD collaboration: H. Fukaya ${ }^{*, a}$, S. Aoki ${ }^{b, c}$, S. Hashimoto ${ }^{d, e}$, T. Kaneko ${ }^{d, e}$, \\ H. Matsufuru ${ }^{d}$, J. Noaki ${ }^{d}$, K. Ogawa ${ }^{f}$ T. Onogi $^{g}$ and N. Yamada ${ }^{d, e}$ \\ ${ }^{a}$ Theoretical Physics Laboratory, RIKEN, Wako 351-0198, Japan' Graduate School of Pure and \\ Applied Sciences, University of Tsukuba, Tsukuba 305-8571, Japan \\ ${ }^{c}$ Riken BNL Research Center, Brookhaven National Laboratory, Upton, NY 11973, USA \\ ${ }^{d}$ High Energy Accelerator Research Organization (KEK), Tsukuba 305-0801, Japan \\ ${ }^{e}$ School of High Energy Accelerator Science, the Graduate University for Advanced Studies \\ (Sokendai), Tsukuba 305-0801, Japan \\ ${ }^{f}$ Physics Department and Center for Theoretical Sciences, National Taiwan University, Taipei, \\ 10617, Taiwan \\ g Yukawa Institute for Theoretical Physics, Kyoto University, Kyoto 606-8502, Japan
}

\begin{abstract}
We calculate the meson correlators in the $\varepsilon$-regime of two-flavor QCD. On a $16^{3} \times 32$ lattice with $a \sim 0.11 \mathrm{fm}$, the lattice simulations are performed with the dynamical overlap fermions. We reduce the sea quark mass down to $\sim 3 \mathrm{MeV}$ and the valence quark masses are taken in the range $1-4 \mathrm{MeV}$. The meson correlators in various channels are compared with the predictions of (partially quenched) chiral perturbation theory (ChPT). Including the NLO order of the $\varepsilon$ expansion, we extract the leading-order low energy constants of ChPT, the pion decay constant $F$ and the chiral condensate $\Sigma$, as $F=87.3(5.5) \mathrm{MeV}$ and $\Sigma^{\overline{\mathrm{MS}}}=[237.8(4.0) \mathrm{MeV}]^{3}$.
\end{abstract}

The XXV International Symposium on Lattice Field Theory

July 30-4 August 2007

Regensburg, Germany

\footnotetext{
${ }^{*}$ Speaker.

†E-mail: hfukaya@riken.jp
} 


\section{Introduction}

Determination of the leading-order parameters of chiral perturbation theory (ChPT), i.e. the chiral condensate $\Sigma$ and the pion decay constant $F$, is the first goal for lattice QCD in establishing the link between QCD and ChPT. This is not an easy task, because the large volume limit must be taken before taking the chiral limit. Both limits require enormous computational cost.

Recently, another approach is getting popular, that is the lattice calculation in the $\varepsilon$-regime. Here, one reduces the quark mass very close to the chiral limit at a fixed volume $V$. Of particular interest is the region where the pion correlation length, or the inverse pion mass $1 / m_{\pi}$, overshoots the size of the box $L$. In this regime, the finite volume effect becomes prominent, but it can be treated in a systematic way within ChPT as an expansion in a new parameter $\varepsilon$ [1, 2, 3], which has the scale $m_{\pi} / \Lambda_{\text {cut }} \sim p^{2} / \Lambda_{\text {cut }}^{2} \sim \varepsilon^{2}$, where $p$ denotes the pion momentum and $\Lambda_{\text {cut }}$ is the cutoff of ChPT. Zero-momentum mode of pion is treated non-perturbatively at the leading order of the $\varepsilon$-expansion. With this expansion, precise predictions for the volume and topological charge dependences of the quark condensate, meson correlators, etc. are obtained in terms of the low-energy constants in the infinite volume.

Simulating lattice QCD in the $\varepsilon$-regime has potential advantages. Computational cost can be kept manageable by staying in a small box, when reducing the quark mass until the chiral extrapolation becomes unnecessary. The remarkable topological charge and quark mass dependences of the meson correlation functions, for instance, in the $\varepsilon$-regime are helpful to identify the correspondence between QCD and ChPT. These nice properties have already been exploited in the quenched QCD studies [4]. In such works the overlap-Dirac operator [5] is used, since any tiny violation of chiral symmetry may be amplified in the $\varepsilon$-regime.

A recent work of the JLQCD collaboration [6] extended the study in the $\varepsilon$-regime to unquenched QCD. (See also [7] for smaller scale works.) On a $16^{3} \times 32$ lattice at the lattice spacing $a \sim 0.11 \mathrm{fm}$ (determined from $r_{0} \sim 0.49 \mathrm{fm}$ ) we have generated 460 configurations with two-flavor dynamical overlap quarks and Iwasaki gauge action. We carried out a simulation at an extremely light sea quark mass $m \sim 3 \mathrm{MeV}$, which is within the $\varepsilon$-regime. Comparing the Dirac spectrum with the predictions of chiral Random Matrix Theory, we extracted the value of $\Sigma$ at the leading order of the $\varepsilon$-expansion as $\Sigma^{\overline{\mathrm{MS}}}(2 \mathrm{GeV})=[251(7)(11) \mathrm{MeV}]^{3}$, where the second error is an estimate of the systematic error due to the NLO effects of the $\varepsilon$-expansion.

In this work, we use the same configurations in the $\varepsilon$-regime to calculate the meson correlators in various channels. Analytic predictions of ChPT for various channels are known to the next-toleading order of the $\varepsilon$-expansion [9, 10]. Their extensions to the partially quenched ChPT have also become available recently [11]. We compare these ChPT predictions with the lattice data to extract $\Sigma$ and $F$ to the NLO accuracy.

\section{2. (Partially quenched) chiral perturbation theory in the $\varepsilon$-regime at fixed topology}

First, we briefly review the results for the meson correlators calculated within (partially quen -ched) chiral perturbation theory. See [9, 10, 11] for details. Here we consider $N_{v}$ valence quarks with a mass $m_{v}$ and $N_{f}=2$ sea quarks with a mass $m_{s}$, both in the $\varepsilon$-regime. 
As a fundamental building block of this section, let us define the partially quenched zero-mode partition function which consists of two physical quarks (of which mass is $m_{s}$ ) and one valence quark (with $m_{v}$ ) and 1 bosonic quark (with $m_{b}$ ), at a fixed topology $v$.

$$
\mathscr{Z}_{v}^{\mathrm{PQ}}\left(\mu_{b} \mid \mu_{v}, \mu_{s}\right) \equiv \frac{1}{\left(\mu_{s}^{2}-\mu_{v}^{2}\right)^{2}} \operatorname{det}\left(\begin{array}{cccc}
K_{v}\left(\mu_{b}\right) & I_{v}\left(\mu_{v}\right) & I_{v}\left(\mu_{s}\right) & I_{v-1}\left(\mu_{s}\right) / \mu_{s} \\
-\mu_{b} K_{v+1}\left(\mu_{b}\right) & \mu_{v} I_{v+1}\left(\mu_{v}\right) & \mu_{s} I_{v+1}\left(\mu_{s}\right) & I_{v}\left(\mu_{s}\right) \\
\mu_{b}^{2} K_{v+2}\left(\mu_{b}\right) & \mu_{v}^{2} I_{v+2}\left(\mu_{v}\right) & \mu_{s}^{2} I_{v+2}\left(\mu_{s}\right) & \mu_{s} I_{v+1}\left(\mu_{s}\right) \\
-\mu_{b}^{3} K_{v+3}\left(\mu_{b}\right) & \mu_{v}^{3} I_{v+3}\left(\mu_{v}\right) & \mu_{s}^{3} I_{v+3}\left(\mu_{s}\right) & \mu_{s}^{2} I_{v+2}\left(\mu_{s}\right)
\end{array}\right),
$$

where $\mu_{b}=m_{b} \Sigma V, \mu_{v}=m_{v} \Sigma V$ and $\mu_{s}=m_{s} \Sigma V . K_{v}$ 's and $I_{v}$ 's are the modified Bessel functions. Note that in the limit $\mu_{b} \rightarrow \mu_{v}$, it reproduces the $N_{f}=2$ full theory partition function

$$
\mathscr{Z}_{v}^{\mathrm{PQ}}\left(\mu_{v} \mid \mu_{v}, \mu_{s}\right)=\mathscr{Z}_{v}^{\text {full }}\left(\mu_{s}\right) \equiv \operatorname{det}\left(\begin{array}{cc}
I_{v}\left(\mu_{s}\right) & I_{v-1}\left(\mu_{s}\right) / \mu_{s} \\
\mu_{s} I_{v+1}\left(\mu_{s}\right) & I_{v}\left(\mu_{s}\right)
\end{array}\right) .
$$

The partially quenched chiral condensate at finite $V$ and $v$ is defined by

$$
\frac{\Sigma_{v}^{\mathrm{PQ}}\left(\mu_{v}, \mu_{s}\right)}{\Sigma} \equiv-\lim _{\mu_{b} \rightarrow \mu_{v}} \frac{\partial}{\partial \mu_{b}} \ln \mathscr{Z}_{v}^{\mathrm{PQ}}\left(\mu_{b} \mid \mu_{v}, \mu_{s}\right) .
$$

It is not difficult to see that in the $\mu_{v} \rightarrow \mu_{s}$ limit, the partially quenched condensate becomes the one in the full theory, $\frac{\Sigma_{v}^{\mathrm{PQ}}\left(\mu_{s}, \mu_{s}\right)}{\Sigma}=\frac{\Sigma_{v}^{\mathrm{f} u l}\left(\mu_{s}\right)}{\Sigma} \equiv \frac{1}{2} \frac{\partial}{\partial \mu_{s}} \ln \mathscr{Z}_{v}^{\text {full }}\left(\mu_{s}\right)$. In the following, we also use the double derivative of the condensate, defined by

$$
\frac{\Delta \Sigma_{v}^{\mathrm{PQ}}\left(\mu_{v}, \mu_{s}\right)}{\Sigma} \equiv \frac{\lim _{\mu_{b} \rightarrow \mu_{v}} \partial_{\mu_{b}} \partial_{\mu_{v}} \mathscr{Z}_{v}^{\mathrm{PQ}}\left(\mu_{b} \mid \mu_{v}, \mu_{s}\right)}{\mathscr{Z}_{v}^{\text {full }}\left(\mu_{s}\right)}
$$

First, we present the correlation functions of the flavored pseudo-scalar and scalar operators, $P^{a}(x)=\bar{q}(x) \tau^{a} \gamma_{5} q(x)$ and $S^{a}(x)=\bar{q}(x) \tau^{a} q(x)$, where $\tau^{a}$ denotes the generator of $S U\left(N_{v}\right)$ group which the valence quark field $q(x)$ belongs to. For these correlators, the partially quenched expression at fixed topology is known to $\mathscr{O}\left(\varepsilon^{2}\right)$ (no sum over $a$ ) [11]:

$$
\begin{aligned}
C_{P}(t) \equiv & \int d^{3} x\left\langle P^{a}(x) P^{a}(0)\right\rangle=\frac{1}{2} \frac{L^{3} \sum_{\text {eff }}^{2}}{\mu_{v}^{\text {eff }}} \frac{\Sigma_{v}^{\mathrm{PQ}}\left(\mu_{v}^{\text {eff }}, \mu_{s}^{\text {eff }}\right)}{\Sigma}-\frac{1}{2}\left[\frac{2 \Sigma^{2}}{F^{2}} \frac{\Delta \Sigma_{v}^{\mathrm{PQ}}\left(\mu_{v}, \mu_{s}\right)}{\Sigma}\right. \\
& \left.+\frac{\Sigma^{2}}{F^{2}} \frac{\partial_{\mu_{v}} \Sigma_{v}^{\mathrm{PQ}}\left(\mu_{v}, \mu_{s}\right)}{\Sigma}-\frac{\Sigma^{2}}{F^{2}} \frac{4}{\mu_{v}^{2}-\mu_{s}^{2}}\left(\frac{\mu_{v} \Sigma_{v}^{\mathrm{PQ}}\left(\mu_{v}, \mu_{s}\right)}{\Sigma}-\frac{\mu_{s} \Sigma_{v}^{\mathrm{full}}\left(\mu_{s}\right)}{\Sigma}\right)\right] h_{1}(t / T), \\
C_{S}(t) \equiv & \int d^{3} x\left\langle S^{a}(x) S^{a}(0)\right\rangle=\frac{L^{3} \Sigma_{\text {eff }}^{2}}{2} \frac{\partial_{\mu_{v}} \Sigma_{v}^{\mathrm{PQ}}\left(\mu_{v}^{\text {eff }}, \mu_{s}^{\text {eff }}\right)}{\Sigma}-\frac{1}{2}\left[\frac{2 \Sigma^{2}}{F^{2}} \frac{v^{2}}{\mu_{v}^{2}}\right. \\
& \left.+\frac{\Sigma^{2}}{F^{2}} \frac{1}{\mu_{v}} \frac{\Sigma_{v}^{\mathrm{PQ}}\left(\mu_{v}, \mu_{s}\right)}{\Sigma}-\frac{\Sigma^{2}}{F^{2}} \frac{4}{\mu_{v}^{2}-\mu_{s}^{2}}\left(\frac{\mu_{v} \Sigma_{v}^{\mathrm{PQ}}\left(\mu_{v}, \mu_{s}\right)}{\Sigma}-\frac{\mu_{s} \Sigma_{v}^{\mathrm{full}}\left(\mu_{s}\right)}{\Sigma}\right)\right] h_{1}(t / T),
\end{aligned}
$$

where $\mu_{i}^{\text {eff }}=m_{i} \Sigma_{\text {eff }} V$, and the $t$ dependence of correlators is represented by a function $h_{1}(t / T) \equiv$ $T\left[(t / T-1 / 2)^{2}-1 / 12\right] / 2$. $\Sigma$ receives one-loop correction as $\Sigma_{\text {eff }}=\Sigma\left(1+3 \beta_{1} / 2 F^{2} V^{1 / 2}\right)$, where $\beta_{1}$ is the so-called shape coefficient. In our numerical study, $\beta_{1}=0.0836$. 
Next, consider the flavored axial-vector and vector operators $A_{0}^{a}(x)=\bar{q}(x) \tau^{a} \gamma_{0} \gamma_{5} q(x)$ and $V_{0}^{a}(x)=\bar{q}(x) \tau^{a} \gamma_{0} q(x)$. Their correlators at $\mathscr{O}\left(\varepsilon^{2}\right)$ in the $N_{f}=2 \mathrm{ChPT}$ are (see [10] for details)

$$
\begin{gathered}
C_{A}(t) \equiv \int d^{3} x\left\langle A_{0}^{a}(x) A_{0}^{a}(0)\right\rangle=-\frac{F^{2}}{2 T}\left\{\mathscr{J}_{+}^{0}+\frac{2}{F^{2}}\left(\frac{\beta_{1}}{V^{1 / 2}} \mathscr{J}_{+}^{0}-\frac{T^{2}}{V} k_{00} \mathscr{J}_{-}^{0}\right)\right. \\
\left.+\frac{4 \mu_{s}}{F^{2}} \frac{\Sigma_{v}^{\text {full }}\left(\mu_{s}\right)}{\Sigma} \frac{T^{2}}{V} h_{1}(t / T)\right\}, \\
C_{V}(t) \equiv \int d^{3} x\left\langle V_{0}^{a}(x) V_{0}^{a}(0)\right\rangle=-\frac{F^{2}}{2 T}\left\{\mathscr{J}_{-}^{0}+\frac{2}{F^{2}}\left(\frac{\beta_{1}}{V^{1 / 2}} \mathscr{J}_{-}^{0}-\frac{T^{2}}{V} k_{00} \mathscr{J}_{+}^{0}\right)\right\},
\end{gathered}
$$

where $k_{00}$ is another numerical factor depending on the shape of the box (in our case, $k_{00}=$ $0.08331) . \mathscr{J}_{ \pm}^{0}$ are defined by

$$
\mathscr{J}_{ \pm}^{0} \equiv \frac{1}{3}\left(3 \mp 1 \pm 2\left[\frac{\partial_{\mu_{s}} \Sigma_{v}^{\mathrm{full}}\left(\mu_{s}^{\mathrm{eff}}\right)}{\Sigma}+2\left(\frac{\Sigma_{v}^{\mathrm{full}}\left(\mu_{s}^{\mathrm{eff}}\right)}{\Sigma}\right)^{2}+\frac{1}{\mu_{s}^{\mathrm{eff}}} \frac{\Sigma_{v}^{\mathrm{full}}\left(\mu_{s}^{\mathrm{eff}}\right)}{\Sigma}-2 \frac{v^{2}}{\left(\mu_{s}^{\mathrm{eff}}\right)^{2}}\right]\right)
$$

Note that $C_{P}(t)$ and $C_{S}(t)$ are sensitive to $\Sigma$ and $F$ enters only at $\mathscr{O}\left(\varepsilon^{2}\right)$. For $C_{A}(t)$ and $C_{V}(t)$, on the other hand, $F$ appears in the leading term and thus can be extracted efficiently.

\section{Lattice simulations}

Here we summarize our numerical set up for the simulations. For other details, see [6]. Our lattice size is $16^{3} \times 32$ and the lattice spacing is determined as $a=0.1111(24)$ fm from the heavy quark potential assuming $r_{0}=0.49 \mathrm{fm}$. We use the overlap fermion [5], of which the Dirac operator with a quark mass $m$ is given by

$$
D(m)=\left(m_{0}+\frac{m}{2}\right)+\left(m_{0}-\frac{m}{2}\right) \gamma_{5} \operatorname{sgn}\left[H_{w}\left(-m_{0}\right)\right],
$$

where $H_{W}\left(-m_{0}\right)=\gamma_{5} D_{W}\left(-m_{0}\right)$ denotes the standard Hermitian Wilson-Dirac operator. We choose $m_{0}=1.6$ throughout this work. (Here and in the following the mass parameters are given in the lattice unit, unless otherwise stated.) For the gauge action, we use the Iwasaki action at $\beta=2.35$ together with an additional determinant factor corresponding to Wilson fermions and associated twisted-mass ghosts [8], which forbids the topology changes along the Monte Carlo updates. In this work, topological charge is fixed to $v=0$. We use the hybrid Monte Carlo (HMC) algorithm. The sign function in (3.1) is approximated by a rational function with Zolotarev's coefficients after projecting out a few lowest-lying eigenmodes.

In this work, we focus on the run at the lightest sea quark mass $m=0.002$, which corresponds to $\sim 3 \mathrm{MeV}$ in the physical unit and the system is well within the $\varepsilon$-regime. We accumulated 4,600 trajectories after discarding 400 trajectories for thermalization. At every 10 trajectories, we calculated the meson correlators in various channels. We take four values of valence quark mass, $m=0.0005,0.001,0.002$, and $0.003(1-4 \mathrm{MeV})$. The inversion of the Dirac operator is performed simultaneously for all the valence quark masses using the multimass solver.

For the meson correlators, the purely low-mode contribution (both quark and anti-quark propagators are represented by the low-lying eigenmodes) is replaced by the low-mode averaged (LMA) one, i.e. the source point is averaged over all lattice sites. We find that LMA with 100 eigenmodes 
improves the statistical signal substantially for the pseudo-scalar and scalar correlators while the improvement is marginal for the axial-vector and vector correlators.

The jackknife bin-size is chosen as 20 , with which the statistical error saturates.

\section{Numerical results}

Now we present the numerical results.

For the axial current we use the local operator $A_{0}^{a}(x)$, which receives finite renormalization. We calculated the renormalization factor using the RI/MOM scheme and obtained $Z_{A}=1.3513(13)$. In the following, we neglect this tiny statistical error for $Z_{A}$.

Fig. 1 (left panel) shows the data for the axial-vector correlator at $m_{v}=m_{s}=0.002$. A two-parameter fit to $(2.7)$ works well $\left(\chi^{2} /\right.$ d.o.f $\left.\sim 0.01\right)$ for the low-mode averaged data (filled squares) as shown by a blue curve. The fitting range is $t \in[12,20]$. From this fit we obtain $\Sigma \sim[260(32) \mathrm{MeV}]^{3}$ and $F \sim 90(6) \mathrm{MeV}$. The statistical error in $\Sigma$ is large $(\sim 30 \%)$ because $\Sigma$ appears only at $\mathscr{O}\left(\varepsilon^{2}\right)$, while $F$ is determined to a good precision.

Next, let us look at the pseudo-scalar channel at $m_{v}=m_{s}=0.002$. Using $F$ obtained via the axial-vector correlator as an input, we fit the pseudo-scalar channel with (2.5) and obtain $\Sigma$ with much better precision. Fig. 1 (right panel) shows the fit curve with a fit range $t \in[12,20]$ $\left(\chi^{2} /\right.$ d.o.f $\left.=0.07\right)$. A two-parameter fit with $F$ as another free parameter does not work because the sensitivity to $F$ is too weak.

To obtain the best result we perform a simultaneous fit to both $P^{a} P^{a}$ and $A_{0}^{a} A_{0}^{a}$ correlators and obtain $\Sigma=[227.6(3.7) \mathrm{MeV}]^{3}$ and $F=87.3(5.6) \mathrm{MeV}$ (or $\sqrt{2} F=123.5(7.9) \mathrm{MeV}$ ), where the statistical error in $a$ is also taken into account. Here the fit range is $t \in[12,20]$ and $\chi^{2} /$ d.o.f $=0.02$. Multiplying the renormalization factor calculated non-perturbatively through the RI/MOM scheme, we obtained the renormalized condensate $\Sigma^{\overline{\mathrm{MS}}}(2 \mathrm{GeV})=[239.8(4.0) \mathrm{MeV}]^{3}$. This value is consistent with our previous result obtained through the Dirac spectrum $\Sigma^{\text {Dirac }}=[251(7)(11) \mathrm{MeV}]^{3}$.

Once the parameters $\Sigma$ and $F$ are determined, there is no additional free parameters at the given order of the $\varepsilon$-expansion, and therefore the comparison provides a stringent test of the lattice data and/or the $\varepsilon$-expansion. For instance, the lattice data for the scalar channel is shown in Fig. 2 (left panel). The curve in the plot is not a fit to the data but a ChPT prediction (2.6). The agreement is remarkable. Furthermore, we also test the consistency with the partially quenched data sets $\left(m_{v} \neq m_{s}\right)$ for the pseudo-scalar channel (Fig. 2 (right panel)). The curves showing the ChPT prediction without free parameters are perfectly consistent with the lattice data.

\section{Discussions}

We have compared the different channels (pseudo-scalar, scalar, axial-vector and vector), partially quenched correlators with four different valence quark masses, and Dirac spectrum, and they are all consistent with each other. For the change of the fitting range within $t_{\min } \in[10,15]$, both $\Sigma$ and $F$ are quite stable (within 1\%) with similar error-bars. For the finite volume correction, the NLO in the $\varepsilon$-expansion, i.e. $\mathscr{O}\left(\varepsilon^{2}\right) \sim p^{2} \sim 1 / L^{2}$, is taken into account within ChPT.

Still, we find some discrepancy in the pion decay constant with the preliminary result $(F=$ 78(3)(1) MeV) obtained in the $p$-regime using the NNLO formula for the chiral extrapolation [12]. 

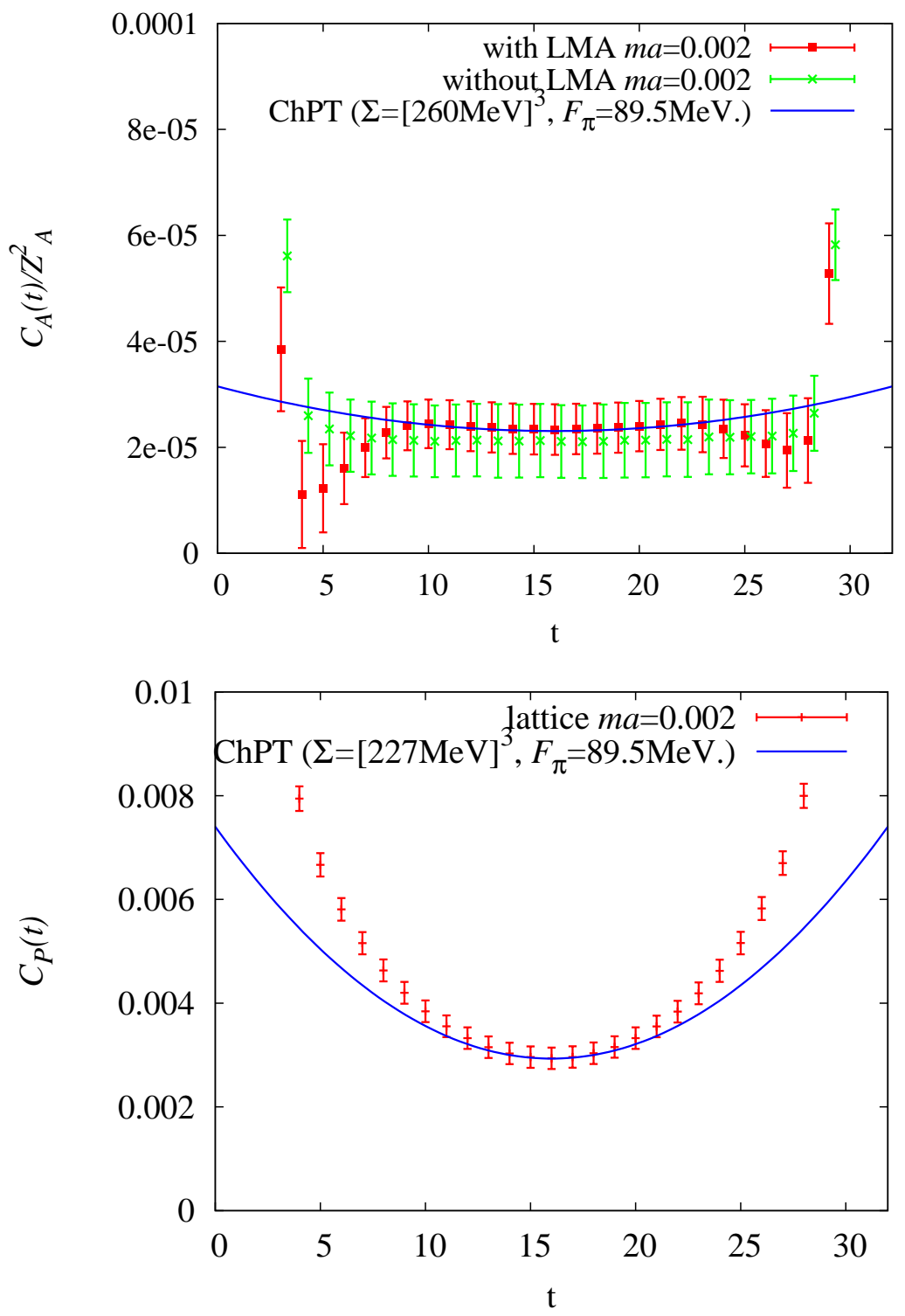

Figure 1: Upper figure: the axial vector correlator in the $\varepsilon$-regime. Filled square symbols denote the lowmode averaged correlator while the crosses are not averaged. The solid curve shows the best fit yielding $\Sigma \sim[260 \mathrm{MeV}]^{3}$ and $\left.F \sim 90 \mathrm{MeV}\right)$. Lower figure: the pseudo-scalar correlator. The solid curve shows the best fit yielding $\Sigma \sim[227 \mathrm{MeV}]^{3}(F=89.5 \mathrm{MeV}$ is given as an input).

(The $p$-regime simulation has been done at a slightly coarser lattice spacing, $a \sim 0.12 \mathrm{fm}$, but we do not expect substantial discretization effect by this small change of $a$.) This may signal some unknown source of systematic error, possibly the higher order contributions in either the $\varepsilon$-expansion or the $p$-expansion.

HF thanks P. H. Damgaard, T. DeGrand and P. Hasenfratz for fruitful discussions. Numerical simulations are performed on Hitachi SR11000 and IBM System Blue Gene Solution at High Energy Accelerator Research Organization (KEK) under a support of its Large Scale Simulation 

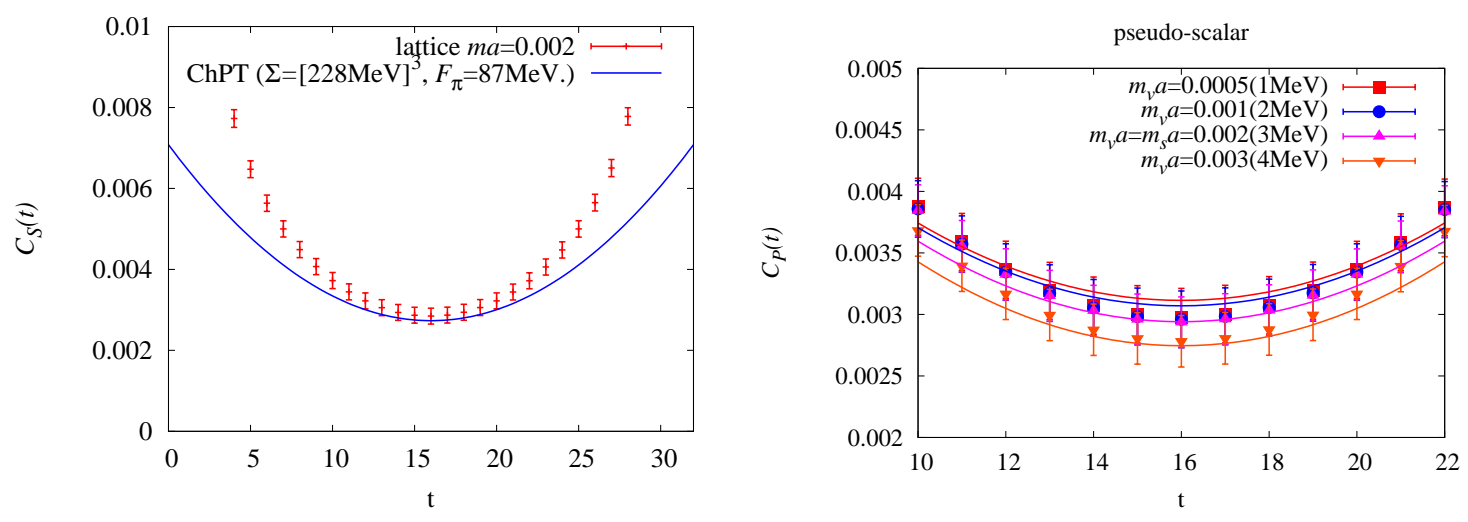

Figure 2: The scalar (left) and the partially quenched pseudoscalar (right) correlators in the $\varepsilon$-regime. The solid curves represent the ChPT prediction with $\Sigma=[227.6 \mathrm{MeV}]^{3}$ and $F=87.3 \mathrm{MeV}$ (No free parameter left).

Program (No. 0716), also in part on NEC SX-8 at YITP, Kyoto University. This work is supported in part by the Grants-in-Aid for Scientific Research from the Ministry of Education, Culture, Sports, Science and Technology. (Nos. 17740171, 18034011, 18340075, 18740167, 18840045, 19540286, 19740160).

\section{References}

[1] J. Gasser and H. Leutwyler, Phys. Lett. B 188, 477 (1987).

[2] F. C. Hansen, Nucl. Phys. B 345, 685 (1990).

[3] P. Hasenfratz and H. Leutwyler, Nucl. Phys. B 343, 241 (1990).

[4] W. Bietenholz, T. Chiarappa, K. Jansen, K. I. Nagai and S. Shcheredin, JHEP 0402, 023 (2004), L. Giusti, P. Hernandez, M. Laine, P. Weisz and H. Wittig, JHEP 0401, 003 (2004), L. Giusti, P. Hernandez, M. Laine, P. Weisz and H. Wittig, JHEP 0404, 013 (2004), H. Fukaya, S. Hashimoto and K. Ogawa, Prog. Theor. Phys. 114, 451 (2005).

[5] H. Neuberger, Phys. Lett. B 417, 141 (1998), H. Neuberger, Phys. Lett. B 427, 353 (1998).

[6] H. Fukaya et al. [JLQCD Collaboration], Phys. Rev. Lett. 98, 172001 (2007), H. Fukaya et al., Phys. Rev. D 76, 054503 (2007).

[7] T. DeGrand, Z. Liu and S. Schaefer, Phys. Rev. D 74, 094504 (2006) [Erratum-ibid. D 74, 099904 (2006)], P. Hasenfratz, D. Hierl, V. Maillart, F. Niedermayer, A. Schafer, C. Weiermann and M. Weingart, arXiv:0707.0071 [hep-lat], T. DeGrand and S. Schaefer, arXiv:0708.1731 [hep-lat].

[8] H. Fukaya, S. Hashimoto, K. I. Ishikawa, T. Kaneko, H. Matsufuru, T. Onogi and N. Yamada [JLQCD Collaboration], Phys. Rev. D 74, 094505 (2006).

[9] P. H. Damgaard, M. C. Diamantini, P. Hernandez and K. Jansen, Nucl. Phys. B 629, 445 (2002).

[10] P. H. Damgaard, P. Hernandez, K. Jansen, M. Laine and L. Lellouch, Nucl. Phys. B 656, 226 (2003).

[11] P. H. Damgaard and H. Fukaya, arXiv:0707.3740 [hep-lat].

[12] JLQCD collaboration (J.Noaki et al.), in these proceedings. 